\title{
Critical Review.
}

\section{THEORETICAL DEDUGTIONS FROM THE PHYSIOLOGY OF THE GEREBRAL GORTEX. By}

\section{DENNY-BROWN, LoNDON.}

IT is now five years since the complete work of Professor Ivan Pavlov on ' conditioned reflexes' first made its appearance in English ${ }^{1}$ and in that time a second volume ${ }^{2}$ has also been published. The far-reaching importance of this original contribution to the physiology of cerebral function cannot be overestimated. Nevertheless, the difficulties inherent in the method and the time and patience required for its performance have resulted in the limitation of first-hand experience to comparatively few workers, with corresponding lack of discussion of the hypotheses which have been put forward to correlate the results of the work. Already writers on cerebral function have attempted to apply the hypotheses of Pavlov to the elucidation of their problems. Therefore it may not be out of place now to review some of these hypotheses. The space of time elapsed since their appearance precludes any such comment from being prejudicial to the reception of Pavlov's work in this country.

In common with many other well-studied nervous phenomena the zonditioned reflexes are still obscure in their essential nature; the following discussion of them indicates a fresh point of view of cortical function.

THE MEANING OF 'REFLEX.'

The word ' reflex' has long had a specialized meaning, denoting automatic fixed inborn responses of the decerebrate nervous system to stimulation of sensory channels. Although, indeed, used by some to describe certain reactions obtained from the intact animal, the word reflex has come to imply assumed identity with the unwilled automatic response obtainable from subcortical nervous structure. In that reflex salivation can be obtained from a decerebrate animal by contact of food with the lining of the mouth, salivation may be reflex. The association of such salivation in response to a stimulus which requires the function of the cerebral cortex for its effect does not necessarily mean that it is then also reflex. Indeed, it is possibly in nature very different from true reflex salivation. The very inflexibility of 
spinal, bulbar and subcortical reflexes is implicit in, and a necessary consequence of, anatomically prearranged fixed nervous channels; but the flexibility of ' conditioned reflexes' points to quite a different mechanism. Flexibility of response to the same stimuli, and habituation to stimuli, appear to be two of the chief characteristics of cerebral nervous function. To identify them with the above fixed responses is to commence by confusing one of the issues of the enquiry.

It is possible that the fixed inborn response may be totally suppressed and replaced by a ' voluntary' or other cortical response. So long as this possibility cannot be eliminated, the identity of one with the other, apart from their final common path, remains a matter for conjecture. The application of the word ' reflex' is best retained for reactions from subcortical levels. The dividing line between conditioned response and reasoned response is vague enough. Pavlov defends his use of the word ' reflex' by emphasising that the reactions of the animal could be described on a physiological basis without postulating motivation. By this bold stroke he establishes an objective approach to his problem. But such use of the word tends to bias the interpretation of the reactions in favour of fixed invariable responses, or to leave the word ' reflex' with no meaning whatever, except response to stimulus. The use of the word by Pavlov to describe the reaction of the dog to the presence of something strange (' investigatory reflex') or to conditions of ' restraint' (' freedom reflex ') or to command (' reflex of slavery'), tends to have the result that each and every movement of an animal is to be called a reflex. Only some of the types of response called ' investigatory reflex'-for example, the investigatory reflex to light (ref. 1, p. 341) and the general auditory reflex to sound (p. 331)-can be obtained from the decorticate animal. The term 'conditioned response' will, therefore, be used hereafter in this discussion instead of, and as equivalent to, Pavlov's term ' conditioned reflex.'

\section{THE MEGHANISM OF FIXATION OF GONDITIONED RESPONSES.}

The essential feature of a conditioned response appears to be the relationship in time between a signal and response-exciting agent (usually a food agent and a corresponding alimentary response). The signal may involve the use of almost any afferent channel of the nervous system, and is a change of environment acquiring its value as a signal simply by virtue of its time relation to the exciting agent. The question of time is important, ' priority' giving the association effect, and immediate priority being more potent than more remote.

For the establishment of the conditional response satisfaction of the nervous state set up is essential. The animal must be satisfied with food after repetition of the signal before the establishment of the signal as a reflex 
stimulus can occur. What does this satisfaction or fulfilment of the train of events mean? Pavlov does not discuss this, although the psychologists have in their field encountered a similar problem for which, in a wider sense, Troland ${ }^{3}$ proposes the term ' retroflex action,' and it is discussed by Lashley ${ }^{4}$ in relation to experiments on learning without, however, any conclusion as to the means by which ' closure' (completion of response) determines retention of habit. To the present writer the importance of fulfilment of the train of events in establishing a conditioned reflex or a habit illustrates a characteristic of cortical processes, and implies a definite kind of mechanism. The last step in the train of events must be the final proprioceptive sensory information reaching the cortex, implying repletion of the hunger need. Hunger, the approach of food indicated by a signal, and the proprioceptive information of fulfilment are the essentials for establishment of the signalsalivation-conditioned response association.

In confirmation of the importance of proprioceptive fulfilment information in cortical processes one may cite the uselessness of the deafferented arm in the monkey as originally demonstrated by Mott and Sherrington ${ }^{5}$ and again more recently in greater detail in experiments by Sherrington ${ }^{6}$ with the writer. Voluntary movement is a poor affair when lacking a running proprioceptive comment, and clinically, in cases of damage to the parietal cerebral cortex, it is obvious that though muscles may be contracted voluntarily, purposive movements are only very gross. Proprioception is in some way essential to cortical function apart from co-ordination of the final motor effect. The conditioned response, in the author's opinion, exemplifies that association. Not only is the information of final fulfilment necessary, but a running commentary, similar to the 'schema' of Head and Holmes?, on the course of the response as well. This is very evident in motor responses, and it should be possible to test in the alimentary conditioned response by appropriate desensitization of tracts of the alimentary canal before attempting the establishment of conditioned responses.

The conditioned response may be described as part of a hunger-signalfulfilment sequence of events, where repetition gives some relative fixity to the sequence. This fixity is reflected in the occurrence of a temporary signalto-preliminary-fulfilment association, the true conditioned response. Upon the variations of this response under various conditions the arguments of Pavlov develop. It is pertinent to enquire as deeply as we can into evidence for these objective phenomena based on cortical processes, and especially to define as closely as possible the functions thereby deduced, at the same time being alert against the besetting tendency to identify the processes with those known in spinal reflexes on the one hand and with psychological conceptions on the other. Too close identification at the outset may only hinder the more rapid progress enabled by a wider, less committal view, 


\section{ATTENTION AND DISTRAGTION.}

The elaborate precautions to isolate the stimulus from other changes of environment by the experimenter with conditioned reflexes illustrate the absolute necessity for avoiding interruption of the experiment. For instance, any extraneous stimulus, a noise or other sensation, concurrent with the stimulus which has been conditioned, interferes with the experiment, and no response may occur. The response is prevented by the extraneous stimulus. There is, however, no evidence that it is actively prevented. To use the word 'inhibit' (' external inhibition') in this sense seems inadvisable. Inhibition in spinal reflexes is an active negative process, an active prevention of excitation, and is set up and persists just as does its corresponding opposite, excitation. Many years of careful work have established the existence of true reflex inhibition. To apply the word to any and every state of affairs where a nervous response fails to occur impedes analysis and is open to confusion.

The reason for the non-occurrence of the conditioned response when an extraneous stimulus interferes with the working of the conditioned signal may possibly be true inhibition; but there is nothing to show that it is not mere passive absence of response.

There is abundant objective evidence that cortical reaction at any one moment is a peak of activity in an otherwise dormant field. In everyday language, attention cannot be divided without impairment of function. To regard interruption of a conditioned reflex by a new, unusual, and therefore functionally more appealing stimulus as a transfer of attention requires no recourse to subjective argument. To a physiologist attention means a concentration of cortical processes upon the most sudden (and least adapted) change in the information from the environment. May it not be that the process which underlies cerebral focus or concentration is, for instance, a reflex link which, by virtue of a strangeness or newness in the environment, is added to otherwise incomplete nervous circuits to allow the easier passage of impulses over the pathways connected to the temporarily most appropriate receptor? For instance, a strange noise will temporarily focus nervous function on hearing to the detriment of visual discrimination. Such a supernumerary labile linkage could not have been considered a few years ago, but the modern hypothesis of subliminal processes in the spinal reflexes ${ }^{8}$ absorbs it without difficulty. Additional subliminal excitation added at any point can, by facilitation, lower the threshold for all reflexes reaching that point, and enable them to produce an effect which otherwise they could not produce. Such an explanation has the merit of being a positive one, for on analogy with the processes of spinal reflexes, it requires only that this or that moiety of the cortex is 'facilitated' by a positive excitation. It necessitates a locus where newness of stimulus can be appreciated, and a 
mechanism whereby the appropriate area of cortex can be lowered in threshold, but we shall see below that such a mechanism may also have a use in other cortical processes, notably in the explanation of sleep.

The process of distraction of a conditioned response therefore becomes a possibility as a purely positive process. A conditioned response is a positive activity; its abeyance may imply merely a shift of the activity elsewhere, and not necessarily a negative, active suppression. The position is clearer perhaps when several conditioned reflexes are interacting. Here it seems simpler and more sound to consider first whether the resultant response be merely the effect of the signal which for purely biological reasons most claims attention, slightly altered maybe by the distracting effect of the others upon the singleness of the attention, rather than to consider possible supposed mutual counterinhibitions interacting and spreading over a cortical receiving mechanism. It may be objected that such a superadded subsidising mechanism, or attentive process, adds to the complication of the supposed mechanism, but Pavlov has to use the 'investigatory reflex' to provide his ' external inhibition,' and its important effects require closer analysis. It seems better here to leave interpretation more open and to call the process ' external inhibition' simply 'external distraction' or ' external interruption.' The process of 'attention' here outlined would appear identical with that described as ' investigatory reflex' by Pavlov, except that 'attention' is assumed to take part in every conditioned response.

When a conditioned response has once been established it reaches a maximum and then gradually fades, so that in time the stimulus produces no effect, even if it be ' reinforced' regularly', and may even become ' inhibitory.' Such fading is regarded by Pavlov as ' functional exhaustion' of the nerve-cells, but not ' autodestruction of the nerve-cells' because reflex inhibition can still be produced by the stimulus. A weakening of the attentive facilitative process would seem here also to give a more satisfactory explanation than that the conditioned response 'develops inhibition,' a negativity which is itself unexplained.

Pavlov finds that a long interval between the signal and the conditioned response, the ' trace reflex,' develops a certain property whereby any other conditioned reflex is depressed or even does not occur during that interval. He supposes that the time interval has ' inhibited' other reflexes. But is this so? It seems obvious that the cerebral cortex is in some way estimating time during the interval. If food has been presented always thirty seconds after the signal then in some way salivation occurs thirty seconds after the signal only because the cortex has in some way been functioning to estimate that time. Here again, without necessarily invoking the activity of mind, there seems to be no real objection to supposing that attention is focussed 
on bodily happenings such as respiration, and the threshold raised for all other conditioned responses because they cannot gain attention. This process could be called an ' internal distraction,' distinct from ' inhibition.' After-effect of such a process reflects the after-effect of the activity underlying it, and is neither for nor against the presence of inhibition.

\section{INHIBITION.}

When a positive conditioned response has been established to one signal, and then another signal repeatedly presented without food (' unreinforced '), this second acquires negative properties. The positive response may be depressed if the positive signal is given just after the negative one. In this case the negative process is rightly called inhibitory, for it exerts actively a suppression of the positive response; and it can suppress other positive responses as well. The evidence, however, indicates that such a negative ' differential inhibition' is not a simple corresponding opposite to the positive conditioned reflex. It is in fact a compound with the positive reflex, and we may suppose that the negative signal has the significance of the words ' not the positive signal.' To endow implied negation with signal value is surely not unphysiological when the receptive organ is one which is adapted for discrimination. The earlier establishment of the positive response is essential; that subsequent negative value is in some way bound to the positive response is illustrated by the way in which a slight external distraction produces once again the earlier positive response, a phenomenon called 'disinhibition ${ }^{10}$. If we suppose the negative superstructure, being always a later addition to the positive response, to require for its effect even more facilitation by attention than does the original positive response, it becomes clear that even a slight distraction can allow a positive response to the negative stimulus. A strong distraction abolishes both reactions. It is absence of proprioceptive repercussion, together with relative likeness to a previously positive signal, which gives the negative signal its value. If there be already more than one established positive signal then an unreinforced signal implies negation of them all and must be engrafted upon each of the conditioned responses. It becomes inhibitory for them all.

If a signal be often encugh repeated without the presentation of the food for which it was conditioned, if in Pavlov's words it be often enough ' unreinforced,' then the conditioned response fades, and eventually becomes ' conditioned inhibition, and the reflex is considered subjected to ' extinction.' Pavlov argues that the process of extinction is not simply fatigue because the response is diminished beyond zero, as measured by the repetitions with reinforcement necessary for recovery. Rupture of anatomical connexions does not take place because the conditioned response will in time recover. He concludes, therefore, that the stimulus becomes - inhibitory.' 
The signal meaning must, however, be here considered, and a reasonable presumption seems to be that, since the whole process depends on an association of two things in time, a recent association of one thing with the lack of a habitual accompaniment must have as much significance as a signal as would its previous association. On this view extinction would be the development of a fresh conditioned response, in this case an active negation, a true inhibitory suppression response, a superstructure to the originally excitatory stimulus. Such experimental inhibitory extinction will in fact recover in time its original positive properties spontaneously, thus revealing the underlying positive process.

\section{THE 'IRRAdIATION' OF GORTIGAL PROGESSES.}

Objective investigation of the capacities of the sense-organs of the dog has advanced much by means of the determination of the least differences of sensation which can develop conditioned responses. How is this differentiation bətween stimuli carried out? A conditioned response when first established is ' generalized,' i.e., a wide group of somewhat similar stimuli all tend to give the same response. The explanation of generalization offered by Pavlov is a spread of excitation in the cortex. His evidence shows that the positive conditioning response has become associated with this wide group of sensory units. The ' spread of excitation,' however, must not here be taken too literally, for it is only the potentially positive linkage which has spread, and not the positive excitatory process itself. Indeed, the writer prefers to interpret the phenomenon of generalization as evidence that the establishment of a positive conditioned response is made only to the general properties of the signal until a negative stimulus is conditioned to eliminate such general properties. For example, a tone of so many vibrations a second at first evokes a response which appears to any similar tone signal. As soon as another stimulus is given a different significance ' differentiation' begins, and the stimuli are active in virtue of their more specific properties. Only stimuli which share uncontrasted properties are now excitants of the reflex. Pavlov calls this ' concentration' of the reflex. In whatever way one may regard this phenomenon it would appear that the cortex does not differentiate finely unless a contrast be offered. The curious mirroring of the signal area from one side of the body to the other found by Anrep ${ }^{11}$ is striking evidence of the generalization of stimulus property. A stimulus from a body point on one side establishes signal value for the same point of the opposite side, until right is differentiated from left. The first tendency of the conditioned response is to make no differentiation, and very close differentiations are most easily made by gradual reduction of contrast. Contrast aids differentiation.

With highly commendable foresight, Pavlov named the receptive side of the conditioned response mechanism the 'analyser,' thus removing all 
implication of anatomical nature from the discussion of sensory functional effects. He speaks of the visual, auditory, tactile and visual analysers as primary divisions of a sensory field. Thus generalization of a stimulus is spoken of as the 'irradiation' of the effect of one particular stimulus to stimuli with related physical properties. In this way tones from similar regions of the scale, or skin points lying close to one another, become potentially endowed with the same effect. It is very necessary to consider in detail just how this ' spread' of effect may occur.

Pavlov supposes that when a certain stimulus reaching an analyser becomes endowed with negative effect in virtue of its relationship to another established negative stimulus, inhibition has spread from the central representative of the one stimulus to that of the other. Inhibition and excitation can, he says, irradiate and concentrate in the analysers. In the sense that the conditioned effect of a stimulus depends on its relation to the effects of similar (therefore nearby) stimuli, inhibitory or excitatory effects may thus be said to be spread in the analyser. It must be pointed out, however, that by analyser Pavlov has defined the central sensorium as a functional plane not to be construed as meaning an anatomical structure, and by ' irradiation' he is construing a widening of response significance in this functional plane. To apply the working of this conception to a layer of cortical nerve-cells is not by any means justified. The inhibition which ' irradiates,' irradiates in the effects produced by analyser mechanism, and to argue that inhibition spreads in the analyser structure itself is without further evidence unjustifiable. To extend the analogy further and speak of 6. . . internal inhibition which is widely irradiated, extending over the whole mass of the hemispheres and involving the lower centres of the brain as well ${ }^{12}$ is not only using the analyser and irradiation within it in a manner which the original definition aims to avoid, but is giving an anatomical basis to properties (such as linear relationship of tone series) which were allowed merely as properties of the analyser because its mechanism was purely functionally considered.

That inhibitory significance spreads from one part of the analyser to another is based on a mass of evidence. The supposition that inhibition therefore spreads from one area of cerebral cortex to another, or from one depth to another depth, rests on no evidence at all.

To return to our own conception of the generalization of stimuli. When first conditioned a stimulus has excitant properties in virtue of its general character only. It will be seen that the irradiation and concentration of the response can equally well be explained on a basis of varying degrees of correspondence, of stimuli one with another in regard to their general properties. 
The specific effect of this or that number of vibrations, or this or that particular area of skin, is acquired only by differentiation, and that in turn. only by offering other distinguishable stimuli. Thus ' a tone' is differentiated as a ' high tone' and then a ' very high tone' and then ' a tone of a particular pitch,' so that the possible number of stimuli fulfilling the essential requirement is narrowed by exclusion of others which are being negatively endowed. Any property of a stimulus may be given the significance of a signal, e.g., its duration, its strength, its rate of repetition, its cessation, or its onset. It is therefore possible for a stimulus to function as a signal in virtue of general or of particular properties. Concentration may surely be looked upon as the differentiation of the particular from the general, and irradiation the converse. We may further extend this hypothesis to deduce that the more contrasted two stimuli may be the less their effect upon each other, for the fewer will be their common identifiable signal properties. There is abundant evidence that this is so. In experiments upon tactile sense ${ }^{13}$, for instance, the points nearest to an inhibitory point suffered the greatest inhibitory effect, and a progressive lessening of effect was observed the further away the application of test stimulus. To call this waning of effect with distance a spread of inhibition or excitation within the analyser leaves the means of spread open for further interpretation, but as used by Pavlov it also gives a distinct impression of a wave of inhibition spreading through layers of cortical cells, an analogy which Pavlov himself tends to further when discussing his hypothesis of sleep. The evidence does not justify diffusinn of any process, still less does it substantiate a hypothetical process spreading beyond the bounds of the part of the cortex concerned with sensory discrimination. It concerns only the development of the capacity to discriminate narrowing or widening cortrast, in quantitative terms of conditioned response linkage.

Dissimilarity is a stimulus-property capable of deciding potency in differentiation. The potential value of a stimulus is related to its similarity to already existing stimuli. If it partakes the properties of a similar established signal Pavlov advances the supposition that the established signal has affected the cortex by positive or negative influence. The fundamental deduction should be that the cortex is unable to differentiate between the two owing to lack of dissimilarity in the stimuli. Closer differentiation is only made by contrasted conditioning. I would like to distinguish between spontaneous differentiation and conditioned differentiation, the former being the property of the cortex of estimating dissimilarity spontaneously, the latter the property of the cortex of estimating dissimilarity by conflicting conditioning. Natural differentiation varies inversely with the multiple of properties common to any two stimuli; the degree of generalization of the effect is the 
degree of generalization of a common property and not the influence of the cortical mechanism for one on that for the other.

When a differentiation is conditioned each step in the approximation of the differentiation is the establishment of two new reflexes, each to a more specific property than the last. The new signal potentialities become associated with more discriminating parts of the analyser; their interaction is on a higher plane. The 'pattern' is changed, but is the same 'pattern' in a fresh place. The plan of potential effect of other related stimuli can - still be predicted from their relationship to the established signals and still rcmains a function of the natural discriminative power of the sensory cortex.

If a stimulus possesses specific properties which it is possible to condition but which the cortex does not differentiate spontaneously these specific properties must always be appreciated by the sensorium. Why are they not differentiated spontaneously if at all? This is the crux of the problem and its solution is the elucidation of the mechanism of the sensory cortex. Pavlov's solution is that such finer discriminations, each reaching a particular point in the analyser, remain impotent until the point is affected by a spread of effective influence from a neighbouring point of established sign. How this spread originates or takes place he does not explain. It has no counterpart in spinal reflexes. Beritoff ${ }^{14}$ states that there is 'general increase in excitability of the whole cortex' during the period of formation of the conditioned response, allowing widespread temporary connexions to be set up between the cutaneous analyser and the motor apparatus. If instead we postulate that the sensory cortex can appreciate finer discriminations by integrative function of sensory correlating neurones, and that this function cccurs only in the presence of strong facilitation provided by the attentive process hypothecated earlier above, then a positive solution of sensory discrimination is found. Pressed to activity by a conflicting environmental situation, the process of attention facilitates a higher discrimination of sensory information and the essential conditioned potential linkage is extended to be attached to this higher plane. Distraction lessens the facilitation so that the earlier low grade differentiation may be restored (disinhibition) $)^{15}$. Pressure of circumstance leads to increased cortical ' vigilance' in the sense proposed by Henry Head ${ }^{16}$.

Thus a positive effect builds superstructure to existing low-grade differentiation.

Contrast in time produces a waning of effect just as much as contrast in space, and the time relations of 'irradiation of inhibition' obtained from the experiments of Kogan and Andréev and others ${ }^{17}$ are readily explained on a basis of increasing time-contrast interacting with space-contrast to give compound differentiations. 


\section{LOGALIZATION OF FUNGTION IN THE GEREBRAL GORTEX.}

The cerebral cortex, even in its earliest beginning (the neopallium of the comparative anatomists), is the meeting ground of all sensory pathways. Pavlov finds by ablation experiments that only finer discriminations in any range of sensation require the area of cerebral cortex commonly accepted as the specific area for that sensation. Thus crude visual discriminations are possible in the frontoparietal cortex, crude auditory discriminations in parts other than the temporal cortex. From experiments upon maze habit learning in rats and monkeys Lashley ${ }^{18}$ concludes that there is a wide generalization of functional effect in the cerebral cortex. Thus these two routes of approach have reached similar conclusions regarding localization of general effects.

A recent excellent review of the question of localization in the cerebral cortex by Hines ${ }^{19}$ summarizes the clinical, anatomical and histological evidence. From the physiological point of view the lack of localization of specific function becomes mure and more apparent.

The motor cortex gives a more direct index of its function than any other, but even here fluctuation in function has for a long time been $\mathbf{k n o w n}^{20}$. More recently Miss Cooper and the author ${ }^{21}$ have found that responses from a ' cortical point' in the motor area show evidence of gross admixture of mutually contradictory functicnal units. Hence fluctuation in function of the point under electrical stimulation may represent only relative selection of one or other element by the spinal neehanism. Nevertheless, a general pattern of localization can be mapped by electrical stimulation and clinicopathological analysis. The present writer would insist that these functional analyses may be, and almost certainly are, but the reflection of a relative concentration of like functional elements. How far scattered the outlying elements may be is a matter for conjecture, for even ' motor' projection cells may not resemble the largest Betz-cells ${ }^{22}$. The response to electrical stimulation is probably only the expression of algebraical summation in the relatively greatest concentrate of functionally similar elements. The microscopically intermingled mosaic of function prevents the artificial stimulus of the experimenter from selecting in its purity any one of its composite functional patterns. The immense practical clinical use of what is already known of motor localization is because disease often affects the motor cortex just as does the electrical stimulus. Clinical evidence points to greater concentration of motor cortical function in man than in animals, but even so localization is far from being absolute in any sense.

So too the calcarine cortex, though it bears a rough pattern corresponding with retinal segments, and though the knowledge of this pattern can be of the greatest practical value in detecting disease by perimetry, has no 
essential attribute of cortical function. As Collier has indicated ${ }^{23}$, it is little more than an entry zone to the cerebral cortex. No discriminative function whatever has been delimited within it, while at least some visual discriminations are possible beyond its limits ${ }^{1,}$.

In such a commingled, heterogeneous organ there must be a discriminative mechanism to which impulses of a primary sensation are relayed on their arrival at cortical levels. This discriminator must be able to extract the utmost significance from any stimulus combination. The site of convergence of one sensation upon another is most simply related to a superposed correlating neurone of the discriminative mechanism. This mechanism may, without anatomical identification, be pictured as a constituent of the whole cerebral cortex wherein, all sensations meeting on equal terms, the ultimate outcome is the result of connexions facilitated by the proprioceptive repercussions which reach the same nexus.

The failure of attempts to localize specific discriminations within this discriminative organ drive one to the provisional conclusion that there is no areal localization within it, and localization in depth gives little satisfaction. The most constant evidence from learning experiments is that quantity of cortical substance determines degree of general efficiency ${ }^{16}$. We have postulated within the organ the effects of a labile facilitating mechanism, attention, against which there is neither functional nor structural evidence. We may presume the discriminative mechanism itself to be a layer of nervecells, but there is no conclusive evidence that it is an identifiable layer such as the middle granule layer, or ' receptive layer' of Bolton".

In the discriminative cortex there is widespread functional representation of proprioception although there is a relatively delimited entry zone in the parietal cortex. Proprioceptive sensation, from its very necessity in purely motor acts, is everywhere in close functional relationship with motor integration. Thus may these two essentials of the conditioned response fuse with a functionally if not structurally homogeneous field.

\section{SLEEP.}

It was argued earlier that a process of attention could, by subsidizing any particular mechanism of the cortex, heighten the activity of that mechanism as against that of all others. When this ' attentive' process is depressed by satiety, or disintegrated by distraction, the conditioned response will not manifest itself. This failure may be only relative, in the sense that it lacks merely the facilitating process. In this way sleep can be explained as the complete suppression or disappearance of the process of attention, lcaving all cortical processes subliminal. If the 'irradiation' of inhibition of Pavlov occurs only as a mechanism of stimulus disccrimination wholly 
dependent upon the contrast property of the stimulus and not as an inhibition of cortical neurones, then the cause of sleep must be looked for in the properties of the very signals initiating the experiments in which it occurs. It is significant that the most potent condition for sleep is that long interval during which in ' trace reflexes' the cerebral cortex is somehow judging time, which it presumably does by attention fixation on some bodily rhythmic function such as respiration. Monotony is the most potent quality of stimulus in predisposition to sleep. The notion of a subcortical (from clinical and physiological evidence possibly thalamic) primitive sensory mechanism concerned in the appreciation of newness or vividness (primitive contrast) of stimulus, and having the power to facilitate the appropriate field of the cortical discriminating mechanism, provides a working hypothesis which accounts for the process of attention in the alert state. The fatigue, or exhaustion, or simple lack of stimulation of such a mechanism accounts for the phenomenon of sleep without the postulation of any active negative process. The transitional states of hypnosis remain as transitional states both on the above hypothesis or on the hypothesis of irradiation of inhibition.

We have already regarded disinhibition, the revelation of the positive element in negative by external distraction, as evidence of the compound nature of true cortical inhibition. This conception requires that the negative superstructure of a conditioned inhibition needs more facilitation from aitention than the original positive response on which it was founded. In this way the hypothesis of functional impairment of a mechanism of attention as an explanation of sleep may be used to explain the excitation immediately preceding sleep $^{25}$ when attention is presumably impaired, and the ' ultraparadoxical' phase succeeding sleep ${ }^{26}$ when grading of attention must also occur. The fact that ' inhibitory reflexes' are at first the only responses to be obtained from an ' analyser' subjected to surgicel damage $^{27}$, and the further evidence that any damage to the cortex impairs all inhibitory processes greatly ${ }^{28}$, suggest that here there is the result of fixation of attention upon a functionless part of the analyser.

\section{FIXATION OF CORTICAL PATHWAYS.}

As to the naturc ci the mechanism of the formation of the transient sensorimotor associations which constitute conditioned reflexes, and probably learning and mind, there is still no direct evidence. The spinal reflexes have no equivalent permanent or long-delayed effects, and their automatism is presumably the reflection of anatomically predetermined connexion. Between conceptions of physiochemical reactions, flow of neurin, interception of neuroglia, or protrusion of dendrites, there is little to choose. The importance of proprioception is manifest, and it is here suggested that a train of events, associated with a running commentary of proprioceptive information and 
finishing with proprioceptive satisfaction of the original cause of attention, in some way lowers the resistance of the whole path. It would seem preferable, by analogy with spinal reflex phenomena and by the speed of performance of cortical reflexes, to suppose that the process is one of lowering of synaptic resistance. The modern conception of reflex mechanisms in the spinal cord ${ }^{8}$ suggests that the lowering of path resistance in cortical linkages is not so much by wearing lower the threshold at the synapse as by the mutually facilitating effects of two paths converging on the same neurone link.

The experimenter with conditioned responses is not dealing with a relatively isolated receptive pathway as is the case in experiments upon spinal reflexes. The receptive cerebral cortex is under constant stimulation, for on it afferent channels bear a constant stream of information. Negation may be implied by change in a particular combination, and be potent in effect. Sequence in time lends differing signal values to the same combinations, so that the apparent paradox that absence of stimulus may itself be a stimulus will occur if an expected sequence be disrupted.

Under these circumstances a cortical process cannot be regarded as a chain, for the whole sensory cortex is welded together with a proprioceptive component into each response. A closer analogy may be that of a weave of cloth, where the individual threads are intermeshed to make a whole, and where for a short time after its unravelling each thread bears a trace of its part of the weave. The motor response is in some way included in the ' weave' although it may not be manifest until late in the process. The experiments of Lashley ${ }^{16}$ show that though the general effect resembles the formation of a pattern, the details of the pattern are not essential.

\section{GONGLUSION.}

In conclusion it may be emphasized that Pavlov has without question gained recognition of the ' conditioned reflexes' as a physiological approach to the problems in the function of the cerebral cortex. That being so it is now very necessary to plead the restriction of the use of such terms as ' inhibition' and ' reflex' to their narrower definition, and to point out the limitations of the direct application of functional conceptions to anatomical structure. In support of my contention that interpretation is not thereby restricted some alternative views are here proposed which provide a working hypothesis to explain cortical phenomena on a positive basis, and one which conflicts less with present conceptions of spinal reflex phenomena.

In order to estimate the presence and nature of nervous processes within the cerebral cortex the amount of effect obtained simply because the cortex fails to make discrimination must first be assessed and discounted. Assessment of degree of difference between stimuli in its finer shades requires some 
subjective reasoning on the part of the assessor; but, for the purpose of analysis of the protocols of most of the conditioning experiments so far published, contrast may be valued purely objectively by plotting the greater or less approximation of purely physical properties of the stimulus, e.g., the number of vibrations a second in a tone, or the distance apart of points of stimulation on a skin surface. Reaction to difference reveals the true analytical function of the cortex.

In the application of the results of the study of conditioned responses to the human cortex the most crucial problem appears to be whether a mental satisfaction can be made equivalent to a physical one. If a ' psychological ' satisfaction can stamp in the train of events preceding it then the application of ' conditioning' to mental habits is clear; but the problem is beyond the scope of experimental physiology. Application of contrast differentiation to problems of mind and behaviour brings all the difficulties of estimating contrast values of abstract stimulus properties objectively. It is precisely at this point that the application of conditioned responses to man meets its greatest difficulty, whatever be the theoretical basis it is attempted to apply.

The establishment and analysis of the conditioned response are by far the most outstanding contribution to the physiology of the cerebral cortex in our time. It is far from the intention of the writer that the criticism and working hypotheses here outlined should in any way, even in appearance, detract from the appreciation of such monumental work. Rather it attempts to give a fuller understanding of Pavlov's work. Between the knowledge of the sensory processes in the cerebral cortex thus gained, and the little definite knowledge that has been gained of the outgoing cortical motor pathway, there remains a complicated nervous mechanism still unexplored.

\section{REFERENCES}

${ }^{1}$ Pavlov, I. P., Conditioned Reflexes: An Investigation of the Physiological Activity of the Cerebral Cortex, 1927.

2 Pavlov, I. P., Lectures on Conditioned Reflexes: Twenty-five Years of Objective Study of the Higher Nervous Activity (Behaviour) of Animals, 1929.

3 Troland, L. T., The Fundamentals of Human Motivation, 1928.

4 Lashley, K. S., The Foundations of Experimental Psychology, edited by Carl Murchison, 1929, 554.

5 Motr, F. W., and Sherrington, C. S., Proc. Roy. Soc., 1895, lvii, 481.

- Sherrington, C. S., Brain, 1931, liv, 1.

7 Head, Henry, and Holmes, Gordon, Brain, 1911-12, xxxiv, 102.

${ }^{8}$ Creed, Denny-Brown, Eccles, Liddell, and Sherrington, Reflex Activity of the Spinal Cord, 1932.

9 Pavlov, Conditioned Reflexes, 1927, 244.

10 id., ibid., 66.

11 id., ibid., 164. 
12 id., ibid., 253.

13 id., ibid., 153.

14 Beritoff, J. S., Brain, 1924, xlvii, 109.

15 Pavlov, Conditioned Reflexes, 1927, 82.

16 Head, Henry, Brit. Jour. Psychol., Gen. Sec., 1923-4, xiv, 126.

17 Pavlov, Conditioned Reflexes, 1927, 207, 210.

18 LASHLEY, K. S., Brain Mechanisms and Intelligence, 1929.

19 Hines, M., Physiol. Revieros, 1929, ix, 462.

20 Brown, T. Graham, and Sherrington, C. S., Proc. Roy. Soc. B., 1912, $\operatorname{lxxxv}, 250$.

${ }^{21}$ Cooper, S., and Denny-Brown, D., Proc. Roy. Soc. B., 1927, cii, 222.

22 Holmes, Gordon, and MAY, W. Page, Brain, 1909, xxxii, 1.

23 Collier, J., Brit. Med. Jour., 1930, i, 55.

24 Bolton, J. S., The Brain in Health and Disease, 1914.

25 Pavlov, Conditioned Reflexes, 1927, 263.

26 id., ibid., 274.

27 id., ibid., 259.

28 id., ibid., 324. 